\title{
Lipoleiomyosarcoma an extremely unusual sarcoma of uterus: A case report
}

\author{
Abhimanyu Jha, Dr Gita Sayami, Deepti Adhikari, \\ Department of Pathology, Tribhuvan University Teaching Hospital
}

\begin{abstract}
Uterine lipoleiomyosarcoma is a heterologous sarcoma composed of variable proportions of malignant lipoblasts histologically corresponding to well differentiated liposarcoma and malignant smooth muscle cells corresponding to leiomyosarcoma. Finding of benign lipomatous component in a typical leiomyoma (lipoleiomyoma) is not an uncommon, however, lipoleiomyosarcoma is an extremely rare malignant tumor and only very few cases have been reported so far. We report a case of lipoleiomyosarcoma of uterine corpus in a postmenopausal woman presenting with lower abdominal pain and abdominal mass. Diagnosis of lipoleiomyosarcoma was confirmed by histopathological examination of hysterectomy specimen. This is the first case of lipoleiomyosarcoma of uterus reported from Nepal.
\end{abstract}

Keywords: Lipoleiomyosarcoma, uterus, histopathology, unusual case.

\section{Introduction}

Leiomyosarcoma (LMS) represents the most common pure uterine sarcoma and comprises slightly over $1 \%$ of all uterine malignancies. ${ }^{1}$ Heterologous sarcoma of uterus containing leiomyosarcoma with a component of liposarcoma, osteogenic sarcoma and rhabdomyosarcoma are extremely rare. ${ }^{2-5}$ Pure liposarcoma (LPS) of uterus although very rare, has been documented in the literature. ${ }^{6}$

Lipoleiomyosarcoma (L-LMS) is a very unusual sarcoma of uterus. Histologically the tumor has a component of liposarcoma composed of malignant immature fat cells "Lipoblasts" in variable proportion and a component of leiomyosarcoma characterized by malignant smooth muscle cells in variable patterns, coagulative necrosis and increased mitosis. The histogenesis of the tumor is not completely clear. The theory of tumor metaplasia is a well recognized phenomenon, is favored in the histogenesis. ${ }^{6}$ The benign counterpart 'the lipoleiomyoma' usually occur in postmenopausal women and it has been postulated that the adipose tissue of a uterine lipomatous tumor is derived from misplaced embryonal mesodermal remnants from totipotential primitive mesenchymal cells with a potential for lipoblastic differentiation.

Uterine lipoleiomyosarcoma is an extremely rare tumor and so far only three cases have been reported in world literature. ${ }^{2-4}$ Although the course of disease and prognosis of liposarcoma and leiomyosarocoma have been very well documented, it is not clear for lipoleiomyosarcoma as only a very few cases have been documented so far.

\section{Case}

A 65 years old postmenopausal lady, Para, presented to Gynecology department of Tribhuvan University Teaching Hospital, with a history of pain abdomen and lower abdominal lump of 4 months duration. An abdominal examination revealed a globular lower abdominal mass corresponding to 22-24 weeks gestational size. Her general physical examination was unremarkable. Abdominal ultrasonogram revealed myoma of uterus with sarcomatous changes. CT-scan of abdomen suggested liposarcoma of uterus. A total abdominal hysterectomy with bilateral salpingoophorectomy was performed. Intraoperatively

Correspondence

Dr Abhimanyu Jha MD.

Lecturer, Department of Pathology. Tribhuvan University Teaching Hospital. Maharajgunj Campus, Maharajgunj, Kathmandu, Nepal, Phone: 9851011684 email:jhaabhimanyu@yahoo.com 
peritoneal cavity showed $100 \mathrm{ml}$ of straw colored fluid which was negative for malignant cells on cytological examination. Omentum did not show any tumor deposits.

Pathological findings: A specimen of uterus with bilateral adnexae was received which showed a well demarcated fleshy intramural growth arising from corpus of the uterus and compressing the endometrial cavity. The growth measured 19X17X14 cm. Cut surface of the tumor showed gray white whorled areas, yellowish areas, focal myxoid changes and showed extensive areas of necrosis \& areas of hemorrhage. Endometrial surface was unremarkable. Growth was limited to the myometrium and no infiltration into surrounding tissue was seen. Bilateral fallopian tubes and ovaries were unremarkable.

Microscopic findings: Microscopic examination of hematoxylin and eosin ( $\mathrm{H} \& \mathrm{E})$ stained multiple sections showed a malignant tumor with variable patterns of growth. A component of malignant smooth muscle cells and a component of malignant adipocytes were seen. The former component showed growth of intersecting bundles and fascicles of malignant smooth muscle cells with large oval to elongated hyperchromatic nuclei showing irregular nuclear membrane and prominent nucleoli. Mitosis was 11 per 10 high power fields, some with atypical features (Figure 1). Extensive areas of coagulative necrosis were seen (Figure 2). The later component showed growth of sheets of malignant adipocytes with vacuolated cytoplasm and highly atypical nuclei (Figure 3). Multinucleated as well as binucleated lipoblasts were also seen. These two components were identified only after extensive sectioning as both the components were lying quite apart and intervening areas showed growth of mature adipocytes as well as mature smooth muscle cells in a

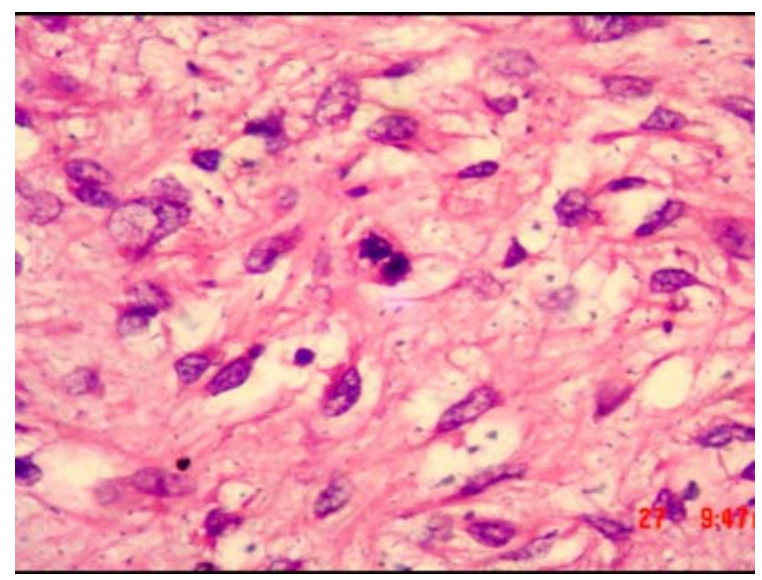

Fig1. Uterine lipoleiomyosarcoma:

Leiomyosarcomatous component, showing atypical spindle cells with highly atypical nuclei and mitosis. (H \& E section: 40X10 magnification) pattern resembling to lipoleiomyoma (Figure 4). Tumor also showed areas of hemorrhage, sclerosis, myxoid changes and thrombosed blood vessels. Lymphovascular invasion was not identified. Endometrium showed atrophic changes and no features of tumor infiltration were seen. Pathological diagnosis of uterine lipoleiomyosarcoma limited to uterus was made.

\section{Discussion}

Lipoleiomyosarcoma, osteogenic sarcoma, rhabdomyosarcoma, and liposarcoma are unusual malignant tumors of the uterus. ${ }^{2-6}$

LMS is the commonest mesenchymal tumor of the uterus and in most of the cases it arises from myometrium. Data from a self referral indigent population at a women's hospital in USA, suggest that pure LMS represent about $1.3 \%$ of all uterine malignancies and about one third of uterine sarcomas. Approximately 1 of every 800 uterine smooth muscle tumor is an LMS, but slightly less than $1 \%$ of women clinically thought to have leiomyoma prove histologically to have LMS ${ }^{8}$ LMS histologically shows malignant smooth muscle cells in intersecting fascicles and sheets with marked nuclear atypia, but high mitotic index and coagulative necrosis are the most important diagnostic criteria in presence of nuclear atypia. The LMS component in present case showed similar findings in present case. Degenerative changes in LMS and symplastic leiomyoma which can mimic liposarcomatous component in $\mathrm{LMS}^{3}$ were ruled out in this case. In presence of degenerative changes mimicking lipoblasts of lipoleiomyosarcoma, typical lipoblastic cells should be searched in the sections. A degenerative change, however, is composed of homogenous population of atypical spindle

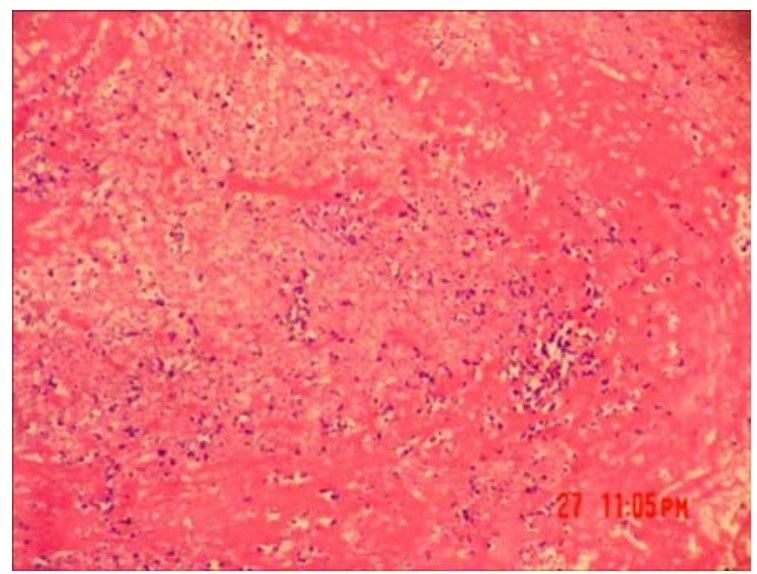

Fig 2. Uterine lipoleiomyosarcoma:

Areas of necrosis. (H \& E section: $10 \times 10$ magnification). 


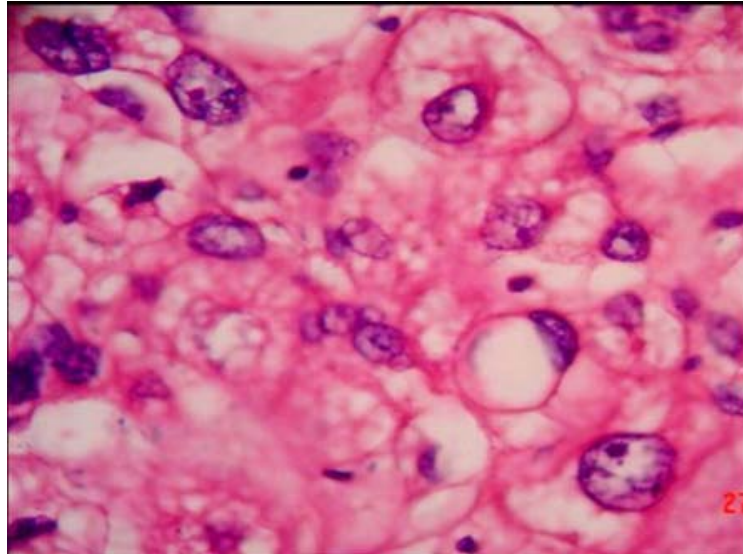

Fig 3. Uterine lipoleiomyosarcoma:

Liposarcomatous component, showing malignant lipoblasts with vacuolated cytoplasm and atypical vesicular nuclei. (H \& E section: $40 \times 10$ magnification)

cells without typical lipoblasts. In present case typical areas of well differentiated LPS was present and no degenerative changes in LMS component was noticed. Atypical (symplastic) leiomyoma is a benign tumor, however, can show atypical epithelioid cells, bizarre cells and multinucleated giant cells which should be differentiated from LMS as well as from liposarcomatous component of L-LMS. But in the case of symplastic leiomyoma there is no coagulative necrosis or increased mitosis to characterize LMS or LLMS. ${ }^{3}$ L-LMS typically shows well differentiated liposarcomatous component in association with LMS as in present case. ${ }^{9}$

In addition to distinguishing L-LMS from pure LMS, it should also be distinguished from pure LPS which have been described in the uterus. ${ }^{6}$ L-LMS should be distinguished from LPS with dedifferentiation having malignant smooth muscle component in dedifferentiated zones but in this case LPS component of the tumor is high grade in contrary to present case. But a leiomyosarcomatous differentiation in LPS containing a well differentiated LPS with intrinsic smooth muscle component is characterized as L-LMS. $^{9}$ In present case dedifferentiated LPS was ruled out as the LPS component showed features of well differentiated LPS. LMS in L-LMS should not be misconstructed as evidence of low grade dedifferentiation as this indicates a more unstable and potentially metastasizing lesion. Folpe and Weiss suggest that L-LMS is a dual lineage sarcoma as evidenced by the fact that the smooth muscle component is often multifocal, not necessarily found in close association with the atypical changes in fat. ${ }^{9}$ In present case LPS and LMS components were not in close association, rather they were separated by

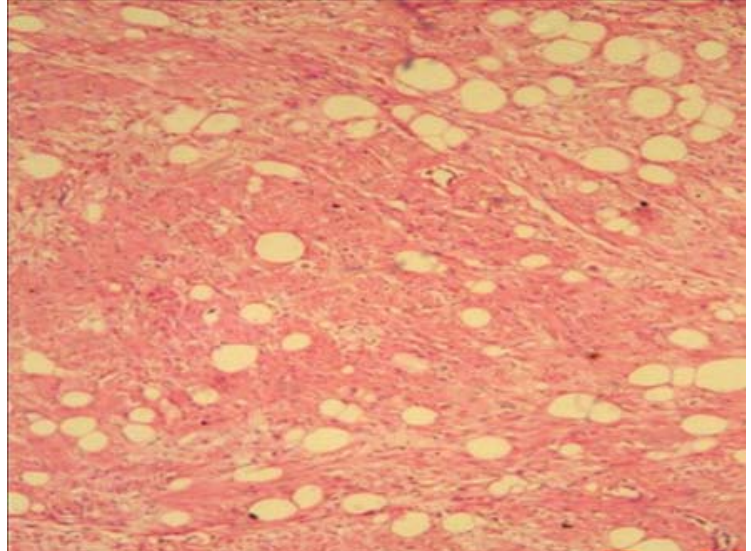

Fig 4. Uterine lipoleiomyosarcoma:

A component of lipoleiyomyoma in the tumor in the areas intervening leiomyosaromatous component and liposarcomatous component. (H \& E section: 10X10 magnification).

a zone of lipoleiomyoma. Uterine lipoleiomyoma a benign mesenchymal tumor composed of mature lipocytes and benign smooth muscle cells have been recognized for many years. The origin of this is postulated to be from misplaced embryonal mesodermal remnants and they have potential for lipoblastic differentiation. $^{7}$

A highly aggressive malignant mixed mullerian tumor (MMT) must be distinguished from L-LMS. ${ }^{3}$ This tumor is not sharply circumscribed tumor as in present case. Histologically MMT shows sarcomatous as well as carcinomatous elements with glandular formation in later. ${ }^{3}$ But in the present case the vacuolated cells fulfilled the criteria of atypical lipoblasts and they were negative for mucin stains, which ruled out carcinomatous component of MMT. It is essential to distinguish L-LMS histologically from benign conditions like angiomyolipoma, spindle cell lipoma, myolipoma and lipoleiomyoma. ${ }^{10}$

A few cases of L-LMS have also been reported in extrauterine sites such as retroperitoneum, paratesticular region, inguinal region, mediastinum, lung, abdomen, popliteal fossa, and in soft tissue. ${ }^{9,10}$ Exact prognosis of heterologous sarcoma of the uterus is not yet established because of rarity of cases. However, these tumors have high metastatic potential. A case of L-LMS metastasizing to vertebra ${ }^{2}$ and a case of rhabdomyosarcoma in association with LMS metastasizing to liver \& lungs ${ }^{5}$ have been documented. This case is unique as the tumor showed all the different patterns of differentiation in same tumor, from mature benign adipocytes and benign smooth muscle cells to multivacuolated atypical lipoblasts and malignant smooth muscle cells. 


\section{Conclusion}

Lipoleiomyosarcoma is a heterologous sarcoma of uterus which is extremely rare and so far only three cases have been reported. It has a potential for distant metastasis, however, exact behavior of the tumor is not known due to paucity of cases. Histologically the tumor should be distinguished from various benign and malignant tumors, especially from dedifferentiated liposarcoma, malignant mixed mullerian tumor, symplastic leiomyoma and anaplastic liposarcoma.

\section{References}

1. Harlow BL, Weiss NS, Lofton S. The epidemiology of sarcomas of the uterus. J Natl Cancer Inst. 1986; 76:399-402.

2. Vakiani M, Mawad J, Talerman A. Heterologous sarcomas of the uterus. Int J Gynecol Pathol. 1982; 1:211-9.

3. Rosenblat Y, Rath-Wolfson L, Rabinerson $\mathrm{D}$, Koren R. Huge uterine tumor in a postmenopausal woman. Arch Pathol Lab Med. 2005; 12:1189-91.

4. Bapat K, Brustein S. Uterine sarcoma with liposarcomatous differentiation: report of a case and review of the literature. Int J Gynaecol Obstet. $1989 ; 28: 71-5$.

5. Shintaku, Masayuki M.D.; Sekiyama, Kentarou M.D Leiomyosarcoma of the Uterus with Focal Rhabdomyosarcomatous Differentiation. Case
Report. International Journal of Gynecological Pathology 2004; 23:188-192.

6. Schneebauer J, Brinninger G, Halabi M, Nagl F. Liposarcoma of the uterus. Gynakol Geburtshilfliche Rundsch. 1996; 36: 90-1

7. Sieinski W. Lipomatous neometaplasia of the uterus. Report of 11 cases with discussion of histogenesis and pathogenesis. Int J Gynecol Pathol. 1989; 8: 357-63.

8. Leibsohn S, d'Ablaing G, Mishell DR Jr, Schlaerth JB. Leiomyosarcoma in a series of hysterectomies performed for presumed uterine leiomyomas. Am J Obstet Gynecol. 1990; 162: 968-74; discussion 974-6.

9. Folpe AL, Weiss SW. Lipoleiomyosarcoma (welldifferentiated liposarcoma with leiomyosarcomatous differentiation): a clinicopathologic study of nine cases including one with dedifferentiation. Am J Surg Pathol. 2002; 26: 742-9

10. Suster S, Wong TY, Moran CA. Sarcomas with combined features of liposarcoma and leiomyosarcoma. Study of two cases of an unusual soft-tissue tumor showing dual lineage differentiation. Am J Surg Pathol. 1993; 17: 905-11.

\section{Acknowledgement}

This case has been presented in IXth Congress of Asia Pacific Association of Societies of Pathologist (APASP), held on November 24-26, 2005, at Kathmandu. 